\title{
Using the PCI-IS Method to Simultaneously Estimate Blood Volume and Quantify Nonvitamin K Antagonist Oral Anticoagulant Concentrations in Dried Blood Spots
}

Ren-Shiang Jhang ${ }^{\dagger}$, Shin-Yi Lin ${ }^{\dagger \dagger}$, Yu-Fong Peng ${ }^{\dagger}$, I-Lin Tsai ${ }^{\S}$, Ya-Ting Lin ${ }^{\dagger}$, Hsiao-Wei Liao ${ }^{\perp}$, Hsi-Chun Chao $^{\dagger}$, Sung-Chun Tang*\#, Ching-Hua Kuo*†, Jiann-Shing Jeng ${ }^{*}$

$\dagger$ School of Pharmacy, College of Medicine, National Taiwan University, Taipei, Taiwan

Department of Pharmacy, National Taiwan University Hospital, Taipei, Taiwan

$\S$ Department of Biochemistry and Molecular Cell Biology, School of Medicine, College of Medicine, Taipei Medical University, Taipei, Taiwan

Faculty of Pharmacy, National Yang-Ming University, Taipei 11221, Taiwan

\# Stroke Center and Department of Neurology, National Taiwan University Hospital

*Corresponding Author

Ching-Hua Kuo

Address: No. 33, Linsen S. Rd., Taipei 100, Taiwan

Tel.: +886.2 .33668766$

E-mail:kuoch@ntu.edu.tw

Sung-Chun Tang

Address: No. 7, Chung-Shan S. Rd., Taipei 100, Taiwan.

Tel.: +886.2.23563279

E-mail: sctang@ntuh.gov.tw 
This SI contains the following:

Section S-1. Sample preparation for volume estimation and evaluation of HCT effect

Section S-2. Bioanalytical method for analysing NOACs in plasma samples

Section S-3. Reported equation for calculating plasma to blood concentration ratio

Table S-1. Parameters of mass spectrometry and retention times for three NOACs and the internal standards.

Table S-2. The estimation accuracy of the spot volume for 7 samples $(n=3)$ using $(\mathrm{A})\left[{ }^{13} \mathrm{C}, d_{3}\right]$-apixaban and (B) $\left[{ }^{13} \mathrm{C}_{6}\right]$-rivaroxaban as the PCI-IS.

Table S-3. The stability of NOACs in DBS samples. The long-term stability of the three NOACs in DBS samples at $\mathrm{RT}, 4^{\circ} \mathrm{C}$ and $-20^{\circ} \mathrm{C}$.

Table S-4. The stability of NOACs in whole blood samples. The stability of the three NOACs in whole blood samples at room temperature (RT) for 2 and 4 hours.

Table S-5. Regression equations of HCT to relative concentration (normalized to plasma concentration) for three NOACs $(n=3)$.

Figure S-1. Comparison of different solvents in extraction solution. The result was calculated as the mean of triplicate injections of $600 \mathrm{ng} \mathrm{mL}^{-1}$ solutions.

Figure S-2. Average \% recovery related bias at $125 \mathrm{ng} \mathrm{mL}^{-1}$ for NOAC quantification obtained at different HCT levels ( $\mathrm{N}=3$ replicates; compared with an HCT of 45\%).

\section{Section S-1. Sample preparation for volume estimation and evaluation of HCT effect}

For the volume estimation experiment, seven different volumes of blood samples were spotted onto DBS cards by pipetting with different blood volumes $(10,15,20,25,30,35 \mu \mathrm{L})$ to test method accuracy (Abstract graphic (B)).

To evaluate HCT effect, the first part of sample preparation was to prepare an artificial blood sample with HCT of $45 \%$. The second part of preparation was to prepare artificial blood samples with different HCT values $(25,35,45,55,65 \%)$ using blood samples with HCT of $45 \%$. Before adjusting the HCT levels, the NOAC standard solution was spiked into the sample. After vortex mixing, the blood sample was kept at room temperature for $5 \mathrm{~min}$ to allow drug distribution. The drug spiked blood sample was subdivided into several samples to prepare samples with different HCT. The blood sample was centrifuged at $15000 \mathrm{rcf}$ for 10 min at $4^{\circ} \mathrm{C}$ to separate the plasma from the red blood cells. The upper plasma layer or the lower red blood cell layer was removed with different amounts to obtain artificial blood samples with specific HCT values $(25,35,45,55,65 \%)$ at a final volume of $120 \mu l$. Fifteen microliters of the blood samples with different HCT values were spotted on Whatman 903 protein saver cards to evaluate HCT effect.

To estimate the contribution of the recovery difference to the HCT effect, we have performed an additional study to estimate whether HCT would cause recovery differences. Same concentration of NOACs 
standard solution was spiked into the blood samples after manual preparation for different HCT levels (25\% to $65 \%$ ). The drug spiked samples were spotted on DBS cards.

\section{Section S-2. Bioanalytical method for analysing NOACs in plasma samples}

The mix working solution of dabigatran, apixaban, and rivaroxaban were prepared at $10,1,0.1 \mu \mathrm{g} \mathrm{mL}^{-1}$ by dilution of the stock solution with methanol. Calibration standards were prepared by diluting working solutions with methanol containing internal standard solutions with $10 \mu \mathrm{g} \mathrm{mL}$, and the final drug concentrations were: $1,2.5,5,10,25,50,100,250,500,750,1000 \mathrm{ng} \mathrm{mL}^{-1}$. All of the solutions were stored at $-20^{\circ} \mathrm{C}$.

For plasma preparation, $100 \mu \mathrm{L}$ of plasma was extracted with $400 \mu \mathrm{L}$ of methanol by shaking for $2 \mathrm{~min}$ at $1000 \mathrm{rpm}$ using a Geno/Grinder 2010 (SPEX® Sample Prep, Metuchen, NJ). The extract was then centrifuged at 15,000 rcf for 5 minutes. Four hundred microliters of supernatant was transferred to a new eppenedorf tube, and the residue was extracted again using the same protocol. The plasma extracts were pooled and dried with a centrifugal vaporizer (Thermo SpeedVac ${ }^{\circledR}$ SPD111V, Waltham, MA, US). The residue was reconstituted with $200 \mu \mathrm{L}$ of methanol followed by Geno/Grinder mixing at 1,000 rpm for 2 min and then centrifuged at 18,000 rpm for $5 \mathrm{~min}$. The supernatant was filtered through a $0.2-\mu \mathrm{m}$ PP membrane filter (RC-4, Sartorius, Göttingen, Germany) and then analyzed by LC-MS/MS.

The analytical performance in terms of extraction recovery, repeatability, accuracy limit of detection, and limit of quantitation were evaluated. Extraction recoveries were evaluated by comparing the peak areas of the pre-spiked samples with the peak area of the post-spiked samples at a NOAC concentration of $50 \mathrm{ng} \mathrm{mL}^{-1}$ $(\mathrm{n}=3)$. Extraction recoveries $(\%)$ for dabigatran, apixaban, and rivaroxaban were $85.2 \pm 0.8,89.1 \pm 1.2$, and $87.7 \pm 1.4$, respectively.

Supplementary Table S-6 shows the linearity, LOQs, and LODs of the three NOACs using the LC-MS platform. The linearity was evaluated using the area ratio of standard-spiked plasma samples with the spiked isotopic labeled internal standards (50 $\mathrm{ng} \mathrm{mL}-1$ of ${ }^{13} \mathrm{C}_{6}$-dabigatran, ${ }^{13} \mathrm{C}_{6}$-apixaban, and ${ }^{13} \mathrm{C}_{6}$-rivaroxaban). To obtain wider linear ranges, two calibration curves were generated and evaluated for the three NOACs. The LOQ and LOD were determined as the ratio of the standard deviation of the response and the slope of the calibration curves with the lower ranges for the three NOACs. LOD and LOQ were calculated using the followed equations.

$$
\begin{aligned}
& L O Q=\frac{10 \sigma}{S} \\
& L O D=\frac{3.3 \sigma}{S}
\end{aligned}
$$

Where $\sigma$ is the standard deviation of the lowest concentration ratio from the calibration curve and $\mathrm{S}$ is the slope of the calibration curve.

Supplementary Table S-7 shows the repeatability and accuracy at three different concentrations for all 
three NOACs using the platform $(n=3)$. The repeatability was lower than $6 \%$ RSD and the accuracy of the method was within the range of 93.3 to $113.3 \%$.

\section{Section S-3 Reported equation for calculating plasma to blood concentration ratio}

$C_{b}=\left[\frac{1-H}{f_{u}}+\mathrm{H} \times p\right] \times f_{u} \times C_{p}$

$\mathrm{C}_{\mathrm{b}}$ : blood concentration

$\mathrm{C}_{\mathrm{p}}$ : plasma concentration

$\mathrm{H}$ : hematocrit, ranging from $0 \sim 1$

$\mathrm{f}_{\mathrm{u}}$ : fraction unbound in plasma (1-protein binding), ranging from $0 \sim 1$

p: erythrocytes-to-unbound plasma concentration ratio

This equation demonstrates the relationship between plasma concentration, blood concentration, and several PK factors. With $\mathrm{f}_{\mathrm{u}}$ and $\mathrm{p}$, we can calculate the ratio of plasma concentration and blood concentration. For dabigatran, $\mathrm{f}_{\mathrm{u}}=65 \%, \mathrm{p}<0.3$. We assumed that normal HCT level is $45 \%$, and we can obtain the ratio: $\mathrm{C}_{\mathrm{p}} / \mathrm{C}_{\mathrm{b}}$ $>1.57$. 
Table S-1. Parameters of mass spectrometry and retention times for three NOACs and the internal standards.

\begin{tabular}{cccccc}
\hline Compound name & $\begin{array}{c}\text { Rt } \\
(\mathrm{min})\end{array}$ & $\begin{array}{c}\text { MRM transition } \\
(\mathrm{m} / \mathrm{z})\end{array}$ & $\begin{array}{c}\text { Type } \\
\text { Fragmentor } \\
(\mathrm{V})\end{array}$ & $\begin{array}{c}\text { Collision } \\
\text { energy }(\mathrm{V})\end{array}$ \\
\hline Dabigatran & \multirow{2}{*}{1.50} & $472.2 \rightarrow 289$ & Quantifier & 125 & 28 \\
Dabigatran & & $472.2 \rightarrow 144$ & Qualifier & 125 & 64 \\
{$\left[{ }^{13} \mathrm{C}_{6}\right]$-dabigatran } & & $478.2 \rightarrow 295.1$ & & 130 & 28 \\
Apixaban & \multirow{2}{*}{2.55} & $460.2 \rightarrow 443.1$ & Quantifier & 170 & 24 \\
Apixaban & & $460.2 \rightarrow 199$ & Qualifier & 170 & 40 \\
{$\left[{ }^{13} \mathrm{C}, d_{3}\right]$-apixaban } & & $464.2 \rightarrow 203.1$ & & 160 & 44 \\
Rivaroxaban & \multirow{2}{*}{2.56} & $436.1 \rightarrow 144.9$ & Quantifier & 160 & 28 \\
Rivaroxaban & & $436.1 \rightarrow 72.9$ & Qualifier & 160 & 70 \\
{$\left[{ }^{13} \mathrm{C}_{6}\right]$-rivaroxaban } & & $442.1 \rightarrow 144.9$ & & 165 & 32 \\
\hline
\end{tabular}


Table S-2. The estimation accuracy of the spot volume for 7 samples $(n=3)$ using $(\mathrm{A})\left[{ }^{13} \mathrm{C}, d_{3}\right]$-apixaban and (B) $\left[{ }^{13} \mathrm{C}_{6}\right]$-rivaroxaban as the PCI-IS.

(A)

\begin{tabular}{ccccccccc}
\hline \multirow{2}{*}{$\begin{array}{c}\text { Volume } \\
(\mu \mathrm{L})\end{array}$} & Sample 1 & Sample 2 & Sample 3 & Sample 4 & Sample 5 & Sample 6 & Sample 7 & RSD\% \\
\cline { 2 - 9 } & $81.6 \pm 3.6$ & $102.2 \pm 8.1$ & $120.9 \pm 16.9$ & $108.6 \pm 6.3$ & $100.4 \pm 6.2$ & $89.6 \pm 2.3$ & $84.9 \pm 4.6$ & 14.2 \\
10 & $89.5 \pm 8.2$ & $116.1 \pm 2.9$ & $113.2 \pm 7.0$ & $111.0 \pm 2.2$ & $117.3 \pm 3.4$ & $90.8 \pm 3.3$ & $99.0 \pm 5.0$ & 11.3 \\
15 & $96.8 \pm 6.1$ & $117.2 \pm 4.9$ & $123.8 \pm 12.4$ & $108.2 \pm 0.4$ & $103.0 \pm 8.5$ & $98.8 \pm 6.9$ & $107.5 \pm 5.0$ & 9.0 \\
20 & $100.7 \pm 9.7$ & $102.0 \pm 3.7$ & $104.4 \pm 9.7$ & $113.3 \pm 11.4$ & $101.0 \pm 7.9$ & $83.1 \pm 8.4$ & $102.8 \pm 5.6$ & 8.9 \\
25 & $89.5 \pm 13.8$ & $98.1 \pm 10.2$ & $95.4 \pm 12.4$ & $93.8 \pm 9.5$ & $92.2 \pm 3.7$ & $82.8 \pm 6.5$ & $100.8 \pm 14.8$ & 6.3 \\
30 & $86.8 \pm 5.9$ & $87.4 \pm 4.8$ & $89.1 \pm 5.7$ & $96.9 \pm 2.9$ & $86.8 \pm 6.0$ & $80.3 \pm 5.9$ & $81.7 \pm 1.0$ & 6.2 \\
35 & & & & & & & \\
\hline
\end{tabular}

(B)

\begin{tabular}{ccccccccc}
\hline \multirow{2}{*}{$\begin{array}{c}\text { Volume } \\
(\mu \mathrm{L})\end{array}$} & Sample 1 & Sample 2 & Sample 3 & Sample 4 & Sample 5 & Sample 6 & Sample 7 & RSD\% \\
\cline { 2 - 9 } & $107.2 \pm 14.7$ & $110.5 \pm 8.2$ & $119.5 \pm 19$ & $119.8 \pm 10.2$ & $116.3 \pm 2.7$ & $97.5 \pm 7.8$ & $98.2 \pm 11.7$ & 8.6 \\
10 & $102.6 \pm 8.1$ & $108.3 \pm 3.5$ & $102.3 \pm 7.0$ & $100.7 \pm 5.3$ & $114.1 \pm 4.3$ & $96.9 \pm 3.4$ & $103.2 \pm 6.5$ & 5.4 \\
15 & $108.3 \pm 24.4$ & $118.7 \pm 8.5$ & $119.4 \pm 3.4$ & $116.7 \pm 5.9$ & $107.3 \pm 4.1$ & $100.6 \pm 3.4$ & $118.0 \pm 5.5$ & 6.5 \\
20 & $95.9 \pm 11.6$ & $108.4 \pm 6.0$ & $113.4 \pm 3.6$ & $101.1 \pm 11.0$ & $103.4 \pm 0.9$ & $87.2 \pm 7.3$ & $95.1 \pm 0.8$ & 8.7 \\
25 & $87.1 \pm 10.1$ & $89.0 \pm 2.8$ & $90.7 \pm 4.1$ & $90.7 \pm 4.4$ & $100.0 \pm 8.4$ & $91.7 \pm 9.0$ & $94.5 \pm 2.3$ & 4.6 \\
30 & $87.8 \pm 6.4$ & $93.9 \pm 11.8$ & $91.0 \pm 6.7$ & $91.5 \pm 2.2$ & $88.0 \pm 9.4$ & $86.1 \pm 5.8$ & $90.2 \pm 2.0$ & 3.0 \\
35 & \multicolumn{7}{c}{$\left.{ }^{13} \mathrm{C}_{6}\right]$-rivaroxaban as the PCI-IS } \\
\hline
\end{tabular}


Table S-3. The stability of NOACs in DBS samples. The long-term stability of the three NOACs in DBS samples at $\mathrm{RT}, 4^{\circ} \mathrm{C}$ and $-20^{\circ} \mathrm{C}$.

\begin{tabular}{|c|c|c|c|c|c|}
\hline \multirow{2}{*}{\multicolumn{2}{|c|}{ Analyte }} & \multicolumn{2}{|c|}{$\begin{array}{c}7 \text { days } \\
(\%, n=3)\end{array}$} & \multicolumn{2}{|c|}{$\begin{array}{c}30 \text { days } \\
(\%, \mathrm{n}=3)\end{array}$} \\
\hline & & Low & High & Low & High \\
\hline \multirow[t]{3}{*}{ Rivaroxaban $^{\mathrm{a}}$} & RT & $102.1 \pm 6.8$ & $102.5 \pm 3.9$ & $98.0 \pm 4.7$ & $98.8 \pm 1.6$ \\
\hline & $4^{\circ} \mathrm{C}$ & $97.6 \pm 1.2$ & $101.0 \pm 2.7$ & $102.2 \pm 2.0$ & $97.7 \pm 0.9$ \\
\hline & $-20^{\circ} \mathrm{C}$ & $101.8 \pm 2.2$ & $98.9 \pm 0.9$ & $106.4 \pm 3.9$ & $95.1 \pm 2.6$ \\
\hline \multirow[t]{3}{*}{ Apixaban ${ }^{b}$} & RT & $95.5 \pm 3.0$ & $96.9 \pm 2.8$ & $109.6 \pm 6.0$ & $104.3 \pm 6.4$ \\
\hline & $4^{\circ} \mathrm{C}$ & $96.3 \pm 3.1$ & $94.6 \pm 1.7$ & $100.9 \pm 6.0$ & $107.5 \pm 4.9$ \\
\hline & $-20^{\circ} \mathrm{C}$ & $94.3 \pm 3.9$ & $91.0 \pm 3.1$ & $102.3 \pm 4.1$ & $99.9 \pm 1.7$ \\
\hline \multirow[t]{3}{*}{ Dabigatran $^{\mathrm{b}}$} & RT & $98.6 \pm 2.8$ & $102.4 \pm 3.5$ & $100.9 \pm 2.9$ & $107.4 \pm 3.9$ \\
\hline & $4^{\circ} \mathrm{C}$ & $101.7 \pm 1.7$ & $102.2 \pm 2.7$ & $99.3 \pm 5.1$ & $111.4 \pm 2.0$ \\
\hline & $-20^{\circ} \mathrm{C}$ & $103.8 \pm 3.1$ & $102.1 \pm 3.5$ & $108.3 \pm 6.3$ & $110.0 \pm 4.7$ \\
\hline
\end{tabular}

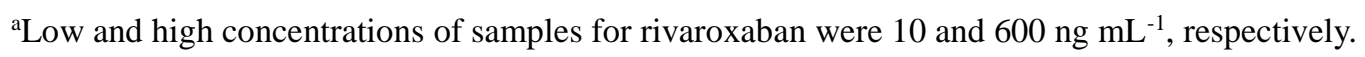

${ }^{\mathrm{b}}$ Apixaban and dabigatran, low and high concentrations were 25 and $600 \mathrm{ng} \mathrm{mL}^{-1}$, respectively.

Table S-4. The stability of NOACs in whole blood samples. The stability of the three NOACs in whole blood samples at room temperature (RT) for 2 and 4 hours.

\begin{tabular}{|c|c|c|c|c|c|}
\hline \multirow[t]{2}{*}{ Stability } & & \multicolumn{2}{|c|}{$\begin{array}{c}2 \text { hours } \\
(\%, n=3)\end{array}$} & \multicolumn{2}{|c|}{$\begin{array}{c}4 \text { hours } \\
(\%, n=3)\end{array}$} \\
\hline & & Low & High & Low & High \\
\hline Rivaroxaban $^{\mathrm{a}}$ & RT & $100.1 \pm 4.7$ & $91.9 \pm 3.0$ & $98.2 \pm 8.1$ & $92.3 \pm 3.7$ \\
\hline Apixaban ${ }^{\mathrm{b}}$ & RT & $94.1 \pm 3.6$ & $90.2 \pm 0.9$ & $95.4 \pm 2.5$ & $89.8 \pm 2.8$ \\
\hline Dabigatran $^{\mathrm{b}}$ & RT & $85.8 \pm 2.5$ & $99.8 \pm 1.2$ & $85.1 \pm 0.1$ & $99.1 \pm 2.1$ \\
\hline
\end{tabular}

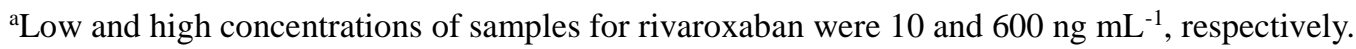

${ }^{\mathrm{b}}$ Apixaban and dabigatran, low and high concentrations were 25 and $600 \mathrm{ng} \mathrm{mL}{ }^{-1}$, respectively. 
Table S-5. Regression equations of HCT to relative concentration (normalized to plasma concentration) for three NOACs $(n=3)$.

\begin{tabular}{ccc}
\hline Analyte & Regression equation & $\begin{array}{c}\text { Pearson correlation } \\
\text { coefficient }\end{array}$ \\
\hline Rivaroxaban & $\mathrm{y}=-54.193 \mathrm{x}+100$ & 0.978 \\
Apixaban & $\mathrm{y}=-70.605 \mathrm{x}+100$ & 0.999 \\
Dabigatran & $\mathrm{y}=-85.147 \mathrm{x}+100$ & 0.999 \\
\hline
\end{tabular}

Table S-6. The linearity, LOQs, and LODs of the three NOACs of the three NOACs using the LC-MS platform $(\mathrm{n}=3)$.

\begin{tabular}{|c|c|c|c|c|}
\hline Analyte & $\begin{array}{l}\text { Linear range } \\
(\mathrm{ng} \mathrm{mL}-1)\end{array}$ & $\begin{array}{c}\text { Linearity } \\
\left(\mathrm{R}^{2}\right)\end{array}$ & $\begin{array}{c}\text { LOD } \\
\left(\mathrm{ng} \mathrm{mL}^{-1}\right)\end{array}$ & $\begin{array}{c}\text { LOQ } \\
\left(\mathrm{ng} \mathrm{mL}^{-1}\right)\end{array}$ \\
\hline Dabigatran & $\begin{array}{c}5-75 \\
75-1000\end{array}$ & $\begin{array}{c}\mathrm{y}=0.0177 x-0.0084 \\
(0.9992) \\
\mathrm{y}=0.0219 x-0.3741 \\
(0.9982)\end{array}$ & 0.22 & 0.67 \\
\hline Apixaban & $\begin{array}{c}5-75 \\
75-750\end{array}$ & $\begin{array}{c}y=0.9068 x-1.5606 \\
(0.9941) \\
y=0.9428 x+0.1974 \\
(0.9959)\end{array}$ & 0.80 & 2.43 \\
\hline Rivaroxaban & $\begin{array}{c}5-50 \\
50-1000\end{array}$ & $\begin{array}{c}\mathrm{y}=0.0158 \mathrm{x}-0.0046 \\
(0.9981) \\
\mathrm{y}=0.0202 \mathrm{x}-0.2111 \\
(0.9999)\end{array}$ & 0.41 & 1.23 \\
\hline
\end{tabular}


Table S-7. The repeatability and accuracy of the three NOACs using the LC-MS platform $(n=3)$.

\begin{tabular}{|c|c|c|c|}
\hline Analyte & $\begin{array}{l}\text { Concentrations } \\
\left(\mathrm{ng} \mathrm{mL}^{-1}\right)\end{array}$ & Repeatability (RSD \%) & $\begin{array}{c}\text { Accuracy } \\
(\%)^{*}\end{array}$ \\
\hline \multirow{4}{*}{ Dabigatran } & 5 & 1.6 & $93.3 \pm 1.3$ \\
\hline & \multirow{2}{*}{$75^{* *}$} & \multirow{2}{*}{0.9} & $99.6 \pm 0.91$ \\
\hline & & & $102.8 \pm 0.7$ \\
\hline & 1000 & 0.9 & $101.9 \pm 1.0$ \\
\hline \multirow{4}{*}{ Apixaban } & 5 & 6.0 & $115.3 \pm 4.9$ \\
\hline & \multirow{2}{*}{$75 * *$} & \multirow{2}{*}{2.3} & $103.3 \pm 2.3$ \\
\hline & & & $96.9 \pm 2.2$ \\
\hline & 750 & 6.4 & $96.6 \pm 0.7$ \\
\hline \multirow{4}{*}{ Rivaroxaban } & 5 & 2.7 & $97.3 \pm 2.5$ \\
\hline & \multirow{2}{*}{$50 * *$} & \multirow{2}{*}{3.4} & $98.8 \pm 2.6$ \\
\hline & & & $96.9 \pm 2.2$ \\
\hline & 1000 & 0.4 & $100.1 \pm 0.47$ \\
\hline
\end{tabular}

* Mean \pm SD

** Evaluated from the two calibration curves, lower range (top) and higher range (bottom). 


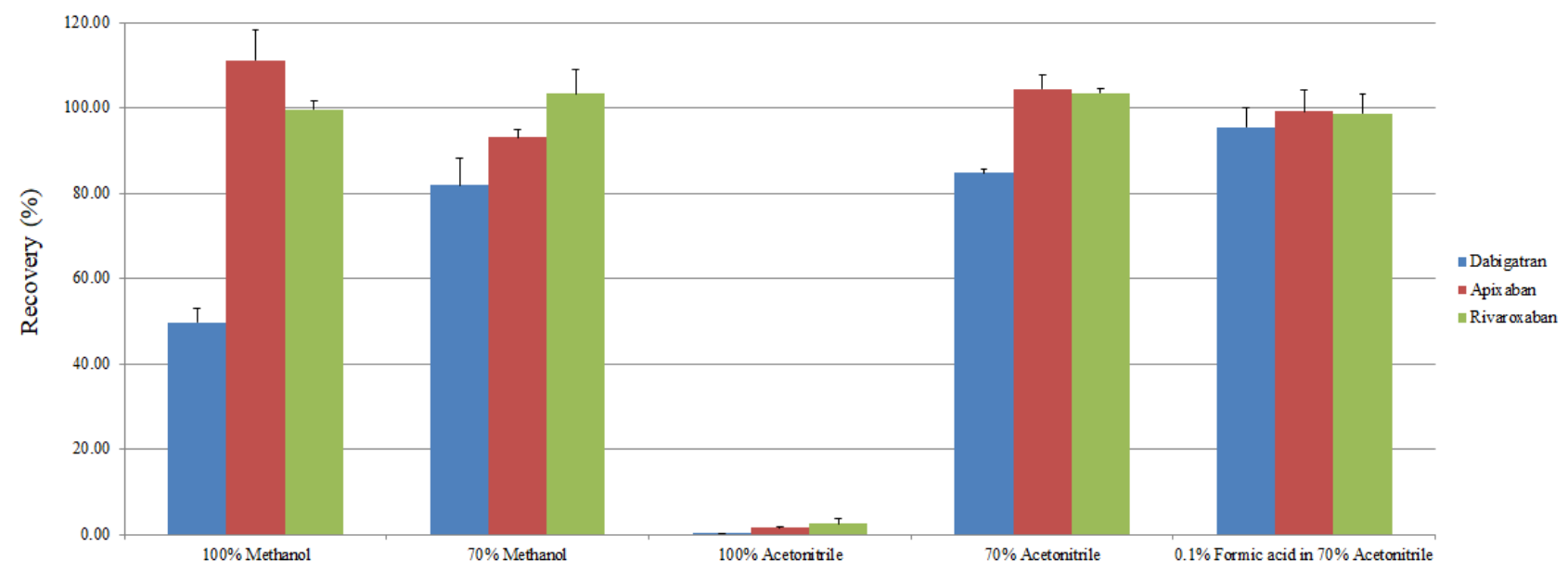

Figure S-1. Comparison of different solvents in extraction solution. The result was calculated as the mean of triplicate injections of $600 \mathrm{ng} \mathrm{mL}^{-1}$ solutions.

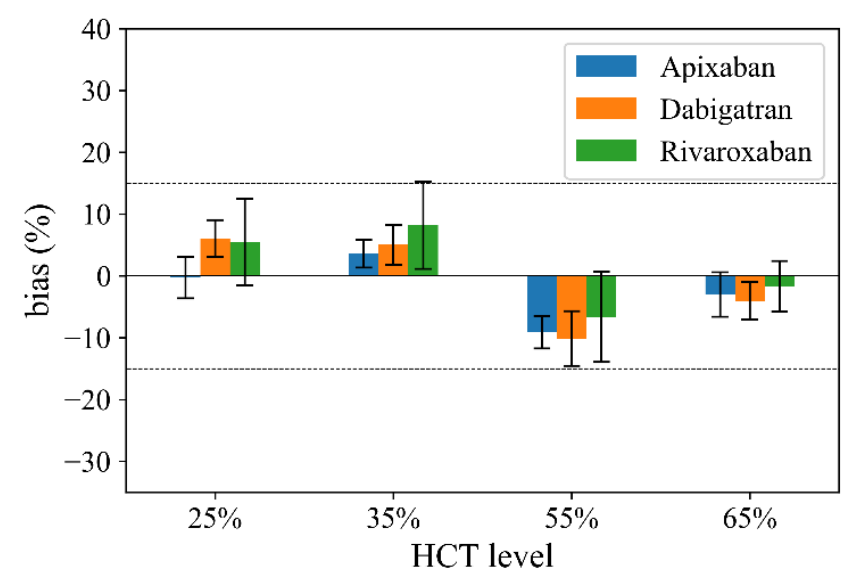

Figure S-2. Average $\%$ bias related bias at $125 \mathrm{ng} \mathrm{mL}^{-1}$ for NOAC quantification obtained at different HCT levels $(\mathrm{N}=3$ replicates; compared with an $\mathrm{HCT}$ of $45 \%)$. 